Innerhalb der ROCKET AF-Studie hatten $52 \%$ einen vorhergehenden vollendeten Schlaganfall $(\mathrm{n}=4.907)$ oder eine TIA $(\mathrm{n}=2.561)$, erhielten also das Medikament zur Sekundärprophylaxe.

Bei den Patienten in der Sekundärprophylaxe lagen die Ereignisraten bei 2,79 unter Rivaroxaban- und 2,96 unter Warfarin-Behandlung; das entspricht einer $6 \%$-igen Risikoreduktion. Für die sekundären Endpunkte wie Hirnblutung, schwerwiegende behindernde Schlaganfälle, tödliche Schlaganfälle, Myokardinfarkte, vaskulärer und nichtvaskulärer Tod ergaben sich keine Unterschiede zwischen Patienten in der Primär- und in der Sekundärprophylaxe. Bei den Blutungskomplikationen zeigte sich bei schwerwiegenden Blutungen kein Unterschied zwischen Rivaroxaban und Warfarin (weder in der Primär- noch in der Sekundärprophylaxe). Bei Blutungen insgesamt ergab sich aber ein Vorteil für Rivaroxaban.

Kommentar: Die Studie ROCKET AF unterscheidet sich von anderen Studien zum Einsatz neuer Antikoagulanzien bei Vorhofflimmern dadurch, dass mehr als die Hälfte aller Patienten bereits eine TIA oder einen vollendeten Schlaganfall. Damit hatten die Patienten ein erhöhtes Risiko. Diese Subgruppe zeigte, dass Rivaroxaban in der Sekundärprophylaxe genauso gut wirkt wie in der Primärprophylaxe, wobei der absolute Therapieeffekt wegen der erhöhten Ereignisraten besser war. Patienten mit bereits stattgehabter TIA und vollendetem Schlaganfall haben auch ein erhöhtes Risiko für intrazerebrale und intrakranielle Blutungen unter Antikoagulation. Dieses Risiko war unter Rivaroxaban signifikant geringer, als unter Warfarin. Damit stellt Rivaroxaban eine wirksame und gegenüber Warfarin sicherere Therapieoption auch in der Sekundärprävention des Schlaganfalls dar.

Prof. Dr. med. Dr. phil. Stefan Evers

Hankey GJ, Patel MR, Stevens SR et al. Rivaroxaban compared with warfarin in patients with atrial fibrillation and previous stroke or transient ischaemic attack: a subgroup analysis of ROCKET AF. Lancet Neurol 2012; 11: 315-22

\title{
Blutdrucksenkung vor und nach Schlaganfall: Wie weit und wie schnell?
}

Die vorliegenden Studie ist eine Metaanalyse aller Studien, die eine normale Blutdrucksenkung mit einer raschen und intensivierten Blutdrucksenkung in der Primär- und Sekundärprävention des Schlaganfalls verglichen haben. Damit nahmen sich die Forscher des Dilemmas an, dass die Leitlinien zur Schlaganfallbehandlung eine Senkung des Blutdrucks unter $140 \mathrm{mmHg}$ empfehlen, eine rasche Blutdrucksenkung aber mehr kardiovaskuläre Ereignisse zur Folge haben kann.

$\mathrm{D}$ as Schlaganfallrisiko steigt mit einem erhöhten systolischen Blutdruck sowohl als primärer als auch als sekundärer Risikofaktor. Aus diesem Grund wird in den Leitlinien zur Schlaganfallbehandlung eine Senkung des Blutdrucks unter systolisch $140 \mathrm{mmHg}$ und bei Patienten mit Diabetes mellitus oder Niereninsuffizienz unter $130 \mathrm{mmHg}$ empfohlen. Auf der anderen Seite gibt es Hinweise dafür, dass eine rasche Senkung des Blutdrucks das Risiko von kardiovaskulären Ereignissen erhöhen kann.

Anzeige

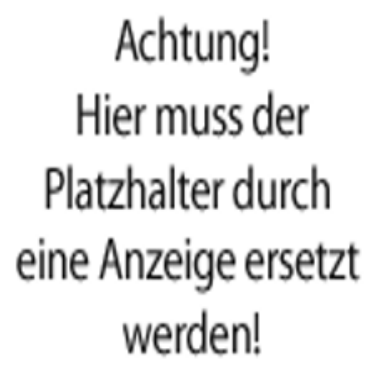

Insgesamt wurden in dieser US-amerikanischen Arbeit elf Studien mit 42.572 Teilnehmern und 794 Schlaganfällen identifiziert. In der Patientengruppe mit intensivierter Blutdrucksenkung wurde ein mittlerer Blutdruckwert von 126,5 $\mathrm{mmHg}$ erreicht. Patienten mit einer normalen Blutdrucksenkung erreichten 132,6 mmHg. Eine intensivierte Senkung des systolischen Blutdrucks führte zu einer Risikominderung für einen Schlaganfall um $20 \%$ und zu einer Risikominderung für schwerwiegende vaskuläre Ereignisse um $9 \%$. Allerdings erzielten diese Risikominderung nur Patienten, die bisher keine kardiovaskulären oder zerebrovaskulären Ereignisse vorweisen konnten.

Kommentar: Diese US-amerikanische Literaturübersicht zeigt, dass Patienten, die bislang keine vaskulären Ereignisse hatten, von einer raschen und intensivierten Blutdrucksenkung auf unter $130 \mathrm{mmHg}$ profitieren können. Allerdings muss dies für Patienten in der Sekundärprävention mit vorherigen Ereignissen relativiert werden. Für diese Patientengruppe scheint es ausreichend, den Blutdruck über Tage mit „normalen" medikamentösen Verfahren auf einen Wert zwischen 130 und $140 \mathrm{mmHg}$ zu reduzieren. Dies ist ein bedeutsames Ergebnis, da eine rasche und intensivierte Senkung des Blutdrucks auch zu Beeinträchtigungen der Lebensqualität und des Wohlbefindens - zumindest für einige Wochen - führen kann. Ein zusätzlicher Nutzen dieser intensivierten Blutdrucksenkung ist somit für diese Patientengruppe nicht belegt.

Prof. Dr. med. Dr. phil. Stefan Evers

Lee M, Saver JL, Hong KS, Hao Q, Ovbiagele $B$. Does achieving an intensive versus usual blood pressure level prevent stroke? Ann Neurol 2012; 71: 133-40 